\title{
Strong Lenses With Single Images
}

\author{
Yiping Shu ${ }^{1}$, Adam S. Bolton ${ }^{1}$, Joel R. Brownstein ${ }^{1}$ and \\ the SLACS Collaborations \\ ${ }^{1}$ Department of Physics and Astronomy, University of Utah, \\ 115 South 1400 East, Salt Lake City, UT 84112, USA \\ email: ypshu@physics.utah.edu
}

SLACS for the masses is the extension of the successful Sloan Lens ACS (SLACS) survey (Bolton et al. 2006, Treu et al. 2006, Koopmans et al. 2006, Gavazzi et al. 2007 and Bolton et al. 2008) but focuses on the lower-mass end of elliptical galaxies (EGs) to yield a more complete strong-lens sample. As to date, 118 out of the 137 proposed candidates have been observed and inspected individually. Among all the targets we have modeled until now, there are:

- 50 grade-A lenses which show clearly lensing features with multiple imaging

- 13 grade-B lenses which have lensing features but no counter-images

The details about image processing can be found in Bolton et al. (2006) and Brownstein et al. (2012). Here we focus particularly on grade-B lenses which have been barely studied due to the absence of counter-images and the difficulty to construct reliable lens models.

For each grade-B lens, we fix all the other lensing parameters to values suggested by the b-spline fit (Bolton et al. 2006) except the Einstein radius $\theta_{\text {Ein }}$ which is gradually varied and fit for the lensed image. Eventually we get a chi-square curve as a function of the trial $\theta_{\text {Ein }}$ (Figure 1.) from which we can infer its upper limit by looking for a point at which the slope of the chi-square curve changes significantly and the fit goes unreasonable after that

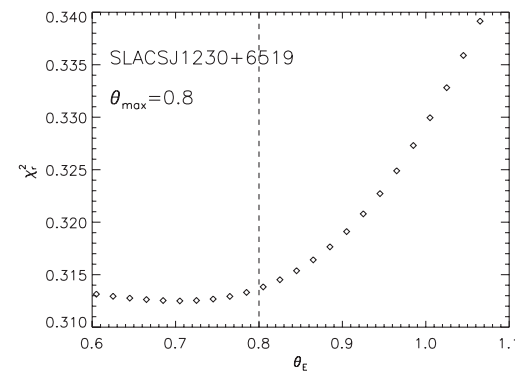

Figure 1. The reduced chi-square curve as a function of the trial Einstein radius for target SLACSJ1230+6519. The dashed line indicates the upper limit of the Einstein radius. point. This set of upper-limit candidates, which are relatively low mass galaxies, extends our understandings of EGs to a wider mass range.

\section{References}

Bolton, A. S., Burles S., Koopmans, L. V. E., Treu, T., Moustakas, L. A., \& Schlegel, D. J. 2006, ApJ, 638, 703

Bolton, A. S., Burles, S., Koopmans, L. V. E., Treu, T., Gavazzi, R., \& Moustakas, L. A. 2008, ApJ, 682, 964

Brownstein, J. R., Bolton, A. S., Schlegel, D. J., Eisenstein, D. J., Kochanek, C. S., Connolly, N., Maraston, C., Pandey, P., Seitz, S., Wake, D. A., Wood-Vasey, W. M., Brinkmann, J., Schneider, D. P., \& Weaver, B. A. 2012, ApJ, 744, 41

Gavazzi, R., Treu, T., Rhodes, J. D., Koopmans, L. V. E., Bolton, A. S., Burles, S., Massey, R. J., \& Moustakas, L. A. 2007, ApJ, 667, 176

Koopmans, L. V. E., Treu, T., Bolton., A. S., Burles, S., \& Moustakas, L. A. 2006, ApJ, 649, 599

Treu, T., Koopmans, L. V. E., Bolton, A. S., Burles, S., \& Moustakas, L. A. 2006, ApJ, 640, 662 